\title{
LDPC-Coded MIMO Receiver Design Over Unknown Fading Channels
}

\author{
Jun Zheng and Bhaskar D. Rao \\ University of California at San Diego \\ Email: juzheng@ucsd.edu, brao@ece.ucsd.edu
}

\begin{abstract}
We consider an LDPC-coded MIMO system composed of $M$ transmit and $N$ receive antennas operating in a flat fading environment. The channel state information is assumed to be unavailable both to the transmitter and the receiver. A soft iterative receiver structure is developed which consists of three main blocks, a soft MIMO detector and two LDPC component soft decoders. Without forming any specific channel estimate, we propose several soft MIMO detectors at the component level that offer an effective tradeoff between complexity and performance. At the structural level, the LDPC-coded MIMO receiver is constructed in a unconventional manner where the soft MIMO detector and LDPC variable node decoder form one super soft-decoding unit, and the LDPC check node decoder forms the other component of the iterative decoding scheme. By exploiting the proposed receiver structure, tractable extrinsic information transfer functions of the component soft decoders are obtained, which further lead to a simple and efficient LDPC code degree profile optimization algorithm with proven global optimality and guaranteed convergence from any initialization. Finally, numerical and simulation results are provided to confirm the advantages of the proposed design approach for the coded system.
\end{abstract}

\section{INTRODUCTION}

Communication systems using multiple antennas at both the transmitter and the receiver have recently received increased attention due to the capability of providing great capacity increases in a wireless fading environment [1]. However, the capacity analysis and MIMO system design is often based on the underlying assumption that the fading channel coefficient between each transmit and receive antenna pair is perfectly known at the receiver. This is not a realistic assumption for most practical communication systems especially in fast fading channels.

For communication systems with unknown channel state information (CSI) at both ends, conventional receivers usually have a two-phase structure, channel estimation using the preset training symbols followed by coherent data detection. Due to the importance of the channel estimator, which directly determines estimation quality and hence the overall system performance, various MIMO channel estimation algorithms have been studied [2] [3]. However, conventional channel estimators form estimates based only on the training symbols, thereby failing to make use of the channel information contained in the received data symbols. Consequently, the twophase model limits the performance and can not approach

This research was supported by CoRe grant No.02-10109 sponsored by Ericsson. the MIMO channel capacity (or the maximum achievable information rate). Possible solutions to the above problem include use of blind source signal separation algorithm [4] [5], MIMO differential modulation [6] [7], and unitary space-time modulation (USTM) [8] [9]. However, none of these schemes can approach the non-coherent MIMO capacity limit due to their sub-optimal code structure, and in the case of USTM, only asymptotic (or the diversity) optimality is achieved in high SNR regimes and the approach suffers from exponential decoding complexity.

In this paper, we focus on the design of practical LDPCcoded MIMO systems employing a soft iterative receiver structure consisting of three component soft decoding blocks, a soft MIMO detector and two soft LDPC component decoders (variable node and check node decoders). At the component level, we propose several soft MIMO detectors, which can generate soft log likelihood ratio (LLR) of each coded bit under the condition of unknown CSIR without forming any explicit channel estimate. At the structural level, the LDPCcoded MIMO receiver is constructed in an unconventional manner where the soft MIMO detector and LDPC variable node decoder form one super soft-decoding unit and the LDPC check node decoder forms the other component of the iterative decoding scheme. Utilizing the receiver structure, tractable extrinsic information transfer functions (EXIT) of the component soft decoders are obtained, which further lead to a simple and efficient LDPC code degree profile optimization algorithm. This algorithm is shown to have global optimality and guaranteed convergence from any initialization, which is in contrast to the sub-optimal manual curve fitting technique proposed in [10].

\section{SySTEM MODEL}

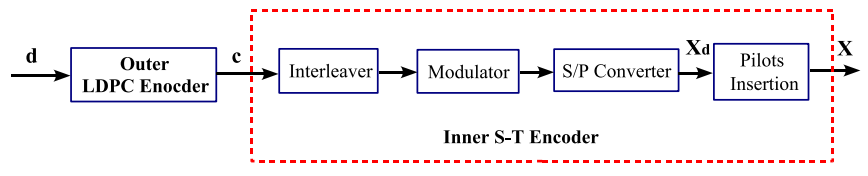

Fig. 1. Transmitter model of LDPC-coded MIMO systems

We consider a MIMO system with $M$ transmit antennas and $N$ receive antennas signaling through a frequency flat fading channel with i.i.d channel coefficients between the transmit 
and receive antenna pairs. As illustrated in Fig. 1, a block of $k$ binary information bits denoted $\mathbf{d}=\left\{d_{1}, \cdots, d_{k}\right\}$ is first encoded by an outer LDPC encoder with code rate $R=k / n$ into a codeword $\mathbf{c}=\left\{c_{1}, \cdots, c_{n}\right\}$ of length $n$. The codeword c is further segmented into $L$ consecutive sub-blocks $\mathbf{C}_{i}$ of length $K$. Each sub-block $\mathbf{C}_{i}$ is then encoded by the inner space-time encoder into a coherent space-time sub-frame $\mathbf{X}_{i}$. This encoder is composed of an interleaver, modulator, serialto-parallel converter, and a pilot insertion operator. Within each sub-frame, the first $p=M T_{\tau}$ symbols are training pilots, followed by $M T_{d}$ data symbols with $T_{d}+T_{\tau}=T$. Hence, the transmitted signal $\mathbf{X}_{i}$ can be stacked and separated into two sub-matrices, i.e.

$$
\mathbf{X}_{i}=\sqrt{\rho / M} \cdot\left[\mathbf{X}_{\tau}^{T}, \mathbf{X}_{d, i}^{T}\right]^{T}
$$

where $\mathbf{X}_{\tau} \in \mathbb{C}^{T_{\tau} \times M}$ are the fixed pilot symbols sent over $T_{\tau}$ time intervals and $\mathbf{X}_{d, i} \in \mathbb{C}^{T_{d} \times M}$ are the information bearing data symbols sent over $T_{d}$ transmission intervals, with the following power constraints,

$$
\operatorname{tr}\left(\mathbf{X}_{\tau}^{H} \cdot \mathbf{X}_{\tau}\right)=M T_{\tau}, \quad E_{\mathbf{X}_{d, i}}\left[\operatorname{tr}\left(\mathbf{X}_{d, i}^{H} \cdot \mathbf{X}_{d, i}\right)\right]=M T_{d}
$$

Therefore, $\rho$ is the average signal to noise ratio (SNR) at each receive antenna. Each element of the transmitted data signal $\mathbf{X}_{d, i}$ comes from a finite complex alphabet $\mathcal{X}$ of size $|\mathcal{X}|$. One entire MIMO codeword $\mathbf{X}$ consists of $l=L T M$ complex symbols, which are transmitted from $M$ transmit antennas and across $L$ consecutive coherent sub-frames of length $T M$ symbols each.

It is assumed that the fading coefficient matrix $\mathbf{H}_{i}$ remains static within each coherent sub-block and varies independently from one sub-block to another. Hence, the signal model can be written as

$$
\mathbf{Y}_{i}=\mathbf{X}_{i} \cdot \mathbf{H}_{i}+\mathbf{w}_{i}, \quad 1 \leq i \leq L,
$$

where $\mathbf{Y}_{i}$ is a $T \times N$ received complex signal matrix, $\mathbf{X}_{i}$ is a $T \times M$ transmitted complex signal matrix, $\mathbf{H}_{i}$ is an $M \times N$ complex channel matrix, and $\mathbf{w}_{i}$ is a $T \times N$ matrix of additive noise. Both matrices $\mathbf{H}_{i}$ and $\mathbf{w}_{i}$ are assumed to have zero mean unit variance independent complex Gaussian entries.

\section{Soft-Input Soft-Output MIMO Detector}

Conventional channel estimators perform estimation only based on the training pilots, thereby failing to make use of the channel information contained in the data symbols. Due to the mismatch between the actual and estimated channel, system performance of the two-phase receiver structure (channel estimation followed by coherent data detection) suffers severe degradation especially in low SNR regimes or fast fading channels.

In this section, several novel MIMO detectors which include the optimal soft MIMO detector as well as two modified sub-optimal detectors are proposed that offer an effective tradeoff between detection complexity and performance. For the sake of simplicity, subscript $i$, denoting the $i^{\text {th }}$ coherent block, is dropped in this section while describing the soft MIMO detection algorithms. To be specific, we denote $\mathbf{X}=$ $\left[\mathbf{X}_{\tau}^{T}, \mathbf{X}_{d}^{T}\right]^{T}, \mathbf{H}$, and $\mathbf{Y}=\left[\mathbf{Y}_{\tau}^{T}, \mathbf{Y}_{d}^{T}\right]^{T}$ as the transmitted signal, channel matrix, and received signal in each coherent block, respectively. Furthermore, sub-matrices $\mathbf{X}_{\tau}, \mathbf{X}_{d}, \mathbf{Y}_{\tau}$, and $\mathbf{Y}_{d}$ have the following structures, i.e.

$$
\begin{aligned}
\mathbf{X}_{\tau}=\left[\mathbf{x}_{\tau, 1}^{T}, \cdots, \mathbf{x}_{\tau, T_{\tau}}^{T}\right]^{T}, & \mathbf{x}_{d}=\left[\mathbf{x}_{d, 1}^{T}, \cdots, \mathbf{x}_{d, T_{d}}^{T}\right]^{T}, \\
\mathbf{Y}_{\tau}=\left[\mathbf{y}_{\tau, 1}^{T}, \cdots, \mathbf{y}_{\tau, T_{\tau}}^{T}\right]^{T}, & \mathbf{Y}_{d}=\left[\mathbf{y}_{d, 1}^{T}, \cdots, \mathbf{y}_{d, T_{d}}^{T}\right]^{T},
\end{aligned}
$$

where $\mathbf{x}_{\tau, k}, \mathbf{x}_{d, k}, \mathbf{y}_{\tau, k}$, and $\mathbf{y}_{d, k}$ represent complex row vectors of size $1 \times M$. Similarly, the binary sub-codeword $\mathbf{C}$ that maps to the transmitted signal $\mathbf{X}$ can also be decomposed into

$$
\mathbf{C}=\left[\mathbf{c}_{1}^{T}, \cdots, \mathbf{c}_{T_{d}}^{T}\right]^{T},\left.\quad \mathbf{c}_{k}\right|_{k=1} ^{T_{d}} \in \mathbb{B}^{1 \times M \cdot \log _{2}|\mathcal{X}|}
$$

where $\mathbb{B}$ is binary set $\{0,1\}$ and each row $\mathbf{c}_{k}$ represents the corresponding binary information that maps to $\mathbf{x}_{d, k}$.

\section{A. Optimal soft MIMO detector}

First, according to the channel model (3), the conditional probability density of the received signal matrix $\mathbf{Y}$ given the transmitted signal matrix $\mathbf{X}$ is given by [11]

$$
p(\mathbf{Y} \mid \mathbf{X})=\frac{\exp \left(-\operatorname{tr}\left\{\left[I_{T}+\mathbf{X X}^{H}\right]^{-1} \cdot \mathbf{Y} \mathbf{Y}^{H}\right\}\right)}{\pi^{T N} \operatorname{det}^{N}\left[I_{T}+\mathbf{X X}^{H}\right]}
$$

It is evident from the above transitional probability that the unknown MIMO channel is actually a memoryless vector channel and hence the optimal MIMO detector does not necessarily need to form a specific channel estimate.

In order to obtain the a posteriori probability of each coded bit, the a priori probability of the input signal matrix $\mathbf{X}$ is first calculated as

$$
p(\mathbf{X})=p\left(\mathbf{X}_{d}\right)=p(\mathbf{C})=\prod_{k=1}^{T_{d}} p\left(\mathbf{c}_{k}\right)=\prod_{k=1}^{T_{d}} \prod_{j=1}^{M \log _{2}|\mathcal{X}|} p\left(c_{k, j}\right)
$$

where each element of matrix $\mathbf{X}_{d}$ is a member of a complex alphabet $\mathcal{X}$ of size $|\mathcal{X}|$, each corresponding to $\log _{2}|\mathcal{X}|$ LDPCcoded bits. Therefore, the log likelihood ratio of each LDPC coded bit is given by

$$
\begin{aligned}
& L_{\mathrm{pos}}\left(c_{k, j}\right)=\log \left(\frac{\Sigma_{\mathbf{X} \in \mathcal{D}_{k, j}^{+}} p(\mathbf{Y} \mid \mathbf{X}) \cdot p(\mathbf{X})}{\Sigma_{\mathbf{X} \in \mathcal{D}_{k, j}^{-}} p(\mathbf{Y} \mid \mathbf{X}) \cdot p(\mathbf{X})}\right), \\
& 1 \leq k \leq T_{d}, \quad 1 \leq j \leq M \cdot \log _{2}|\mathcal{X}|,
\end{aligned}
$$

where $\mathcal{D}_{k, j}^{+}\left(\mathcal{D}_{k, j}^{-}\right)$is the set of $\mathbf{X}$ for which the $(k, j)^{\text {th }}$ bit $c_{k, j}$ of the LDPC coded sub-block $\mathbf{C}$ is " +1 " (" -1 "). Finally, by subtracting the input a priori information from the obtained a posterior log likelihood ratio, the soft extrinsic information of each coded bit is obtained as,

$$
L_{\text {ext }}\left(c_{k, j}\right)=L_{\text {pos }}\left(c_{k, j}\right)-\log \left(\frac{p\left(c_{k, j}=1\right)}{p\left(c_{k, j}=0\right)}\right),
$$


Notice that there is no channel estimation stage in the soft MIMO detector described above, and therefore the proposed detection algorithm does not depend on the unknown channel state $\mathbf{H}$ but only on its underlying statistical distribution.

\section{B. Sub-optimal soft MIMO detector}

The optimal soft MIMO detection algorithm proposed in Section III-A provides the optimal extrinsic LLR values of each coded bit. However, the summation in both the numerator and the denominator of equation (8) consists of $2^{K-1}$ items, with $K\left(=T_{d} M \log _{2}|\mathcal{X}|\right)$ increasing linearly with number of data slots $T_{d}$ (or coherence time $T$ ). It has an unaffordable exponential complexity for practical communication systems, especially when the coherence time $T$ is large. Hence, we propose a sub-optimal MIMO detector in this section with complexity increasing linearly with $T_{d}$.

Instead of performing soft MIMO detection by one operation, we can extract partial extrinsic information by processing only two rows of the data matrix $\mathbf{X}_{d}$ at a time, and then combining different partial extrinsic information to form the final extrinsic LLR. In order to combine information from coded rows $\mathbf{x}_{d, k}$ and $\mathbf{x}_{d, k^{\prime}}$, we first perform the optimal MIMO detection algorithm on the following reduced size sub-coherent block

$\mathbf{X}_{\left[k, k^{\prime}\right]}=\left[\mathbf{X}_{\tau}^{T}, \mathbf{x}_{d, k}^{T}, \mathbf{x}_{d, k^{\prime}}^{T}\right]^{T}, \mathbf{Y}_{\left[k, k^{\prime}\right]}=\left[\mathbf{Y}_{\tau}^{T}, \mathbf{y}_{d, k}^{T}, \mathbf{y}_{d, k^{\prime}}^{T}\right]^{T}$.

Therefore, the partial extrinsic LLR value $L_{\mathrm{ext}, k^{\prime}}\left(c_{k, j}\right)$ of bit $c_{k, j}$ obtained from the a priori information of row $\mathbf{c}_{k}, \mathbf{c}_{k^{\prime}}$, and channel observation $\mathbf{Y}_{\left[k, k^{\prime}\right]}$ is given by

$$
\begin{aligned}
& L_{\text {ext }, k^{\prime}}\left(c_{k, j}\right) \\
& \quad=\log \left(\frac{\Sigma_{\mathbf{X}_{\left[k, k^{\prime}\right]} \in \mathcal{D}_{k, j}^{+}} p\left(\mathbf{Y}_{\left[k, k^{\prime}\right]} \mid \mathbf{X}_{\left[k, k^{\prime}\right]}\right) \cdot p\left(\mathbf{X}_{\left[k, k^{\prime}\right]}\right)}{\Sigma_{\mathbf{X}_{\left[k, k^{\prime}\right]} \in \mathcal{D}_{k, j}^{-}} p\left(\mathbf{Y}_{\left[k, k^{\prime}\right]} \mid \mathbf{X}_{\left[k, k^{\prime}\right]}\right) \cdot p\left(\mathbf{X}_{\left[k, k^{\prime}\right]}\right)}\right) \\
& \quad-\log \left(\frac{p\left(c_{k, j}=1\right)}{p\left(c_{k, j}=0\right)}\right), \quad 1 \leq k, k^{\prime} \leq T_{d}
\end{aligned}
$$

where $\mathcal{D}_{k, j}^{+}\left(\mathcal{D}_{k, j}^{-}\right)$is the set of $\mathbf{X}_{\left[k, k^{\prime}\right]}$ for which bit $c_{k, j}$ is " $+1 "("-1 ")$. By the same reasoning, partial extrinsic information of bit $c_{k, j}$, related to (and contained in) the a priori information of $\mathbf{c}_{k}$ and channel observations $\mathbf{Y}_{\tau}$ and $\mathbf{y}_{d, k}$ can also be obtained by performing optimal detection on the following sub-coherent block

$$
\mathbf{X}_{[k]}=\left[\mathbf{X}_{\tau}^{T}, \mathbf{x}_{d, k}^{T}\right]^{T}, \quad \mathbf{Y}_{[k]}=\left[\mathbf{Y}_{\tau}^{T}, \mathbf{y}_{d, k}^{T}\right]^{T},
$$

with the corresponding extrinsic LLR value given by

$$
\begin{aligned}
L_{\text {ext-p }}\left(c_{k, j}\right) & =\log \left(\frac{\Sigma_{\mathbf{X}_{[k]} \in \mathcal{D}_{k, j}^{+}} p\left(\mathbf{Y}_{[k]} \mid \mathbf{X}_{[k]}\right) \cdot p\left(\mathbf{X}_{[k]}\right)}{\Sigma_{\mathbf{X}_{[k]} \in \mathcal{D}_{k, j}^{-}} p\left(\mathbf{Y}_{[k]} \mid \mathbf{X}_{[k]}\right) \cdot p\left(\mathbf{X}_{[k]}\right)}\right) \\
& -\log \left(\frac{p\left(c_{k, j}=1\right)}{p\left(c_{k, j}=0\right)}\right), \quad 1 \leq k \leq T_{d} . \quad \text { (13) }
\end{aligned}
$$

Having obtained extrinsic information $L_{\mathrm{ext}, k^{\prime}}\left(c_{k, j}\right)$ and $L_{\text {ext-p }}\left(c_{k, j}\right)$, one can obtain by the following substraction,

$$
L_{\text {ext-d, } k^{\prime}}\left(c_{k, j}\right)=L_{\text {ext }, k^{\prime}}\left(c_{k, j}\right)-L_{\text {ext-p }}\left(c_{k, j}\right),
$$

the extrinsic information of bits $c_{k, j}$ extracted solely from the channel observation $\mathbf{y}_{d, k^{\prime}}$ and the a priori information of $\mathbf{c}_{k^{\prime}}$.

In contrast to the situation of perfect channel state information at the receiver (CSIR) where $L_{\text {ext }}\left(c_{k, j}\right)$ only depends on the a priori knowledge of $\mathbf{c}_{k}$ and observation $\mathbf{y}_{d, k}$, a non-zero extrinsic information of $c_{k, j}$ can be obtained from the a priori knowledge of $\mathbf{c}_{k^{\prime}}$ and observation $\mathbf{y}_{k^{\prime}}$ (with $k^{\prime} \neq k$ ) in an unknown MIMO fading environment. An intuitive explanation of above difference can be made by viewing $\mathbf{c}_{k^{\prime}}$ as partially fixed pilots based on the input a priori information. Therefore, better channel knowledge is learned (although no explicit channel estimation exists), which translates into a better a posterior probability of $c_{k, j}$. Hence, a non-zero partial extrinsic information solely from the a priori probability of $\mathbf{c}_{k^{\prime}}$ and the channel observation $\mathbf{y}_{k^{\prime}}$ is obtained.

Due to the assumption that the input a priori information of different bits are independent, all the partial extrinsic information $L_{\text {ext-d, } k^{\prime}}\left(c_{k, j}\right)$ and $L_{\text {ext-p }}\left(c_{k, j}\right)$ can be viewed as being close to independent. The final output extrinsic information $L_{\text {ext }}\left(c_{k, j}\right)$ is obtained by summing all the independent partial extrinsic information obtained from different coded rows $\mathbf{c}_{k^{\prime}}$ and pilot observations, i.e.

$$
\begin{aligned}
& L_{\text {ext }}\left(c_{k, j}\right)=\sum_{\substack{k^{\prime}=1 \\
k^{\prime} \neq k}}^{T_{d}} L_{\text {ext-d, } k^{\prime}}\left(c_{k, j}\right)+L_{\text {ext-p }}\left(c_{k, j}\right) \\
& =\sum_{\substack{k^{\prime}=1 \\
k^{\prime} \neq k}}^{T_{d}} L_{\mathrm{ext}, k^{\prime}}\left(c_{k, j}\right)-\left(T_{d}-2\right) \cdot L_{\mathrm{ext}-\mathrm{p}}\left(c_{k, j}\right),
\end{aligned}
$$

A summation of $2^{2 M \log _{2}|\mathcal{X}|}$ terms is required to extract the partial extrinsic information $L_{\mathrm{ext}, k^{\prime}}\left(c_{k, j}\right)$ in equation (11) and $2^{M \log _{2}|\mathcal{X}|}$ terms for $L_{\text {ext-p }}\left(c_{k, j}\right)$ in equation (13). Therefore, in order to obtain the output soft extrinsic LLR values, a total number of $\left(\left(T_{d}-1\right) \cdot 2^{2 M \log _{2}|\mathcal{X}|}+2^{M \log _{2}|\mathcal{X}|}\right)$ terms of probability summation is required for each coded bit, as opposed to $2^{T_{d} M \log _{2}|\mathcal{X}|}$ terms in the original optimal soft MIMO detector.

\section{Sub-optimal butterfly soft MIMO detector}

Motivated by the fast Fourier transform (FFT) algorithm, we can further reduce the complexity of the soft MIMO detector to $\left(\log _{2} T_{d} \cdot 2^{2 M \log _{2}|\mathcal{X}|}+2^{M \log _{2}|\mathcal{X}|}\right)$ terms of summation per coded bit by using a sub-optimal butterfly MIMO detector structure.

It is first assumed that the number of the data slots $T_{d}=$ $2^{m}$ is power of 2 . If not, we can appropriately zero-pad the transmitted signal matrix $\mathbf{X}$. The sub-optimal butterfly detection algorithm obtains the extrinsic information through a multi-level structure similar to the fast Fourier transform, where the extrinsic information is accumulated from level to level. Specifically, if the partial extrinsic LLR value of coded 
bit $c_{k, j}$ at the $n^{\text {th }}$ level is $L_{\text {ext-d }}^{n}\left(c_{k, j}\right)$, then the extrinsic LLR value of the $(n+1)^{\text {th }}$ level is updated as

$L_{\text {ext-d }}^{n+1}\left(c_{k, j}\right)=L_{\text {ext-d }}^{n}\left(c_{k, j}\right)+\Delta L_{\text {ext-d }}^{n+1}\left(c_{k, j}\right), 0 \leq n \leq m-1$,

where the second term $\Delta L_{\text {ext-d }}^{n+1}\left(c_{k, j}\right)$ of equation (16) represents the additional partial extrinsic information obtained from the information of coded bits $\mathbf{c}_{k^{\prime}}$, with sub-codeword row index $k^{\prime}$ given by

$$
k^{\prime}=\left\{\begin{array}{ll}
k+2^{m-n-1} & \text { if } \quad k\left(\bmod 2^{m-n}\right)<2^{m-n-1} \\
k-2^{m-n-1} & \text { if } k\left(\bmod 2^{m-n}\right) \geq 2^{m-n-1}
\end{array} .\right.
$$

Similar to the extraction algorithm provided in (14), $\Delta L_{\text {ext-d }}^{n+1}\left(c_{k, j}\right)$ is given by the following form

$$
\Delta L_{\text {ext-d }}^{n+1}\left(c_{k, j}\right)=L_{\text {ext }}^{n+1}\left(c_{k, j}\right)-L_{\text {ext-p }}\left(c_{k, j}\right),
$$

where $L_{\text {ext-p }}\left(c_{k, j}\right)$ is given by equation (13), and partial extrinsic information $L_{\mathrm{ext}}^{n+1}\left(c_{k, j}\right)$ is obtained by performing optimal soft MIMO detection on the sub-coherent block $\mathbf{X}_{\left[k, k^{\prime}\right]}$ and $\mathbf{Y}_{\left[k, k^{\prime}\right]}$ with modified input a priori information, i.e.

$$
\begin{aligned}
& L_{\mathrm{ext}}^{n+1}\left(c_{k, j}\right) \\
& =\log \left(\frac{\Sigma_{\mathbf{X}_{\left[k, k^{\prime}\right]} \in \mathcal{D}_{k, j}^{+}} p\left(\mathbf{Y}_{\left[k, k^{\prime}\right]} \mid \mathbf{X}_{\left[k, k^{\prime}\right]}\right) \cdot p_{\mathrm{app}}^{n+1}\left(\mathbf{X}_{\left[k, k^{\prime}\right]}\right)}{\Sigma_{\mathbf{X}_{\left[k, k^{\prime}\right]} \in \mathcal{D}_{k, j}^{-}} p\left(\mathbf{Y}_{\left[k, k^{\prime}\right]} \mid \mathbf{X}_{\left[k, k^{\prime}\right]}\right) \cdot p_{\mathrm{app}}^{n+1}\left(\mathbf{X}_{\left[k, k^{\prime}\right]}\right)}\right) \\
& -\log \left(\frac{p\left(c_{k, j}=1\right)}{p\left(c_{k, j}=0\right)}\right) .
\end{aligned}
$$

Furthermore, the modified a priori probability $p_{\text {app }}^{n+1}\left(\mathbf{X}_{\left[k, k^{\prime}\right]}\right)$ in equation (19) is a combination of the a priori probability of $\mathbf{c}_{k}$ and $\mathbf{c}_{k^{\prime}}$ as well as the $n^{\text {th }}$ level extrinsic information of $\mathbf{c}_{k^{\prime}}$, which can be represented as

$$
p_{\mathrm{app}}^{n+1}\left(\mathbf{X}_{\left[k, k^{\prime}\right]}\right)=\prod_{j=1}^{M \log _{2}|\mathcal{X}|} p\left(c_{k, j}\right) \cdot p\left(c_{k^{\prime}, j}\right) \cdot p_{\mathrm{ext}}^{n}\left(c_{k^{\prime}, j}\right),
$$

where $p_{\mathrm{ext}}^{n}\left(c_{k^{\prime}, j}\right)$ is given by

$$
p_{\text {ext }}^{n}\left(c_{k^{\prime}, j}\right)=\frac{\exp \left(c_{k^{\prime}, j} \cdot L_{\text {ext-d }}^{n}\left(c_{k^{\prime}, j}\right)\right)}{1+\exp \left(L_{\text {ext-d }}^{n}\left(c_{k^{\prime}, j}\right)\right)} .
$$

Therefore, $\Delta L_{\text {ext-d }}^{n+1}\left(c_{k, j}\right)$ can be viewed as the partial extrinsic information obtained solely from the a priori information of $\mathbf{c}_{k^{\prime}}$, channel observation $\mathbf{y}_{k^{\prime}}$, and its extrinsic information at the $n^{\text {th }}$ level.

Starting from the initial condition $L_{\text {ext-d }}^{0}\left(c_{k, j}\right)=0$, the extrinsic information $L_{\text {ext-d }}^{n}\left(c_{k, j}\right)$ of each coded bit is accumulated at each level by absorbing additional partial extrinsic information through the sub-coherent block combining process. The final soft extrinsic LLR value of each coded bit is formed by combining the extrinsic LLR information at the $m^{\text {th }}$ (lowest) level with the extrinsic information obtained from pilot observations, which is given by

$$
L_{\text {ext }}\left(c_{k, j}\right)=L_{\text {ext-d }}^{m}\left(c_{k, j}\right)+L_{\text {ext-p }}\left(c_{k, j}\right) \quad 1 \leq k \leq T_{d} .
$$

\section{Discussion}

Note that both the sub-optimal structure in Section III-B as well as the sub-optimal butterfly MIMO detector in the previous subsection are modifications of the optimal soft MIMO detection algorithm provided in Section III-A. The two suboptimal MIMO detection algorithms provided in Section III-B and III-C have the following structural differences. First, the sub-optimal MIMO detector in Section III-B forms extrinsic information through a linear combining structure, where there are a total of $\left(T_{d}-1\right)$ partial extrinsic information terms (each corresponding to the partial extrinsic LLR obtained from other rows $k^{\prime}$ ); each term is computed by performing optimal detection on the sub-coherent block given by (11)-(14). On the other hand, the sub-optimal butterfly MIMO detector in Section III-C performs data detection by employing a multilevel structure, where the extrinsic information is distributed at succeeding levels until all the input a priori information and the channel observations are combined and exchanged between all different rows.

\section{ReCEIVER Design OF THE CODED MIMO System}

A. New receiver structure of the LDPC-coded MIMO system

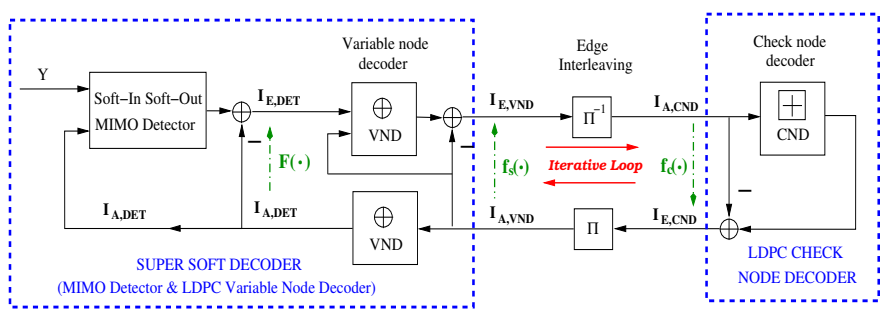

Fig. 2. Receiver structure of LDPC-coded MIMO systems

Conventionally a coded MIMO receiver is obtained by connecting the inner soft MIMO detector and the outer LDPC decoder to form one large iterative decoding loop. The overall MIMO receiver actually consists of two iterative decoding loops. The soft extrinsic information, which describes the uncertainty of each coded bits, is iteratively exchanged in the outer loop between the MIMO detector and LDPC decoder as well as in the inner loop between variable node and check node decoders inside the LDPC decoder.

In this paper, we structure the MIMO receiver differently by combining the soft MIMO detector and LDPC variable node decoder together as a super soft decoder, a form also suggested in [10]. As illustrated in Fig. 2, the decoding loop is formed by exchanging extrinsic information between the super decoder and the LDPC check node decoder iteratively. Compared with the conventional iterative MIMO receiver (named as bit-interleaved coded modulation with iterative decoding (BICM-ID) algorithm), the new receiver structure has two advantages. First, the new receiver structure has only one iterative decoding loop and hence has lower decoding complexity compared to the conventional BICM-ID receiver structure. Second, the proposed structure has the advantage of 
enabling the EXIT function of the soft component decoders to have tractable forms. By fully exploiting the closed form EXIT functions, a simple iterative approach for the LDPC code degree profile optimization with guaranteed convergence and global optimality is proposed in Section IV-B, which is superior to the sub-optimal manual curve fitting technique [12] [10].

\section{B. LDPC code optimization}

Following the methodology given in [10] [12], the EXIT functions of the super MIMO soft decoder (combination of the LDPC variable node decoder and soft MIMO detector) can be obtained as

$$
\begin{array}{r}
I_{\mathrm{E}, \mathrm{VND}}=f_{s}\left(I_{\mathrm{A}, \mathrm{VND}}\right)=\sum_{i=1}^{D_{v}} \lambda_{i} \cdot J\left(\left(d_{v, i}-1\right) \cdot J^{-1}\left(I_{\mathrm{A}, \mathrm{VND}}\right)\right. \\
+J^{-1}\left(\left.F\right|_{\rho}\left(J\left(d_{v, i} \cdot J^{-1}\left(I_{\mathrm{A}, \mathrm{VND}}\right)\right)\right)\right),(23)
\end{array}
$$

where $\lambda_{i}$ is the fraction of the variable nodes having edge degree $d_{v, i}$, and $D_{v}$ is the number of different variable node degrees. The function $J(\cdot)$ is given by

$$
\begin{aligned}
J\left(\sigma_{A}^{2}\right)=\frac{1}{\ln 2}\left(\frac{1}{\sigma_{A}^{2}}-\int_{-\infty}^{\infty}\right. & \frac{\sigma_{A}}{\sqrt{2 \pi}} \ln \cosh (y) \\
& \left.\times \exp \left(-\frac{\left(\sigma_{A}^{2} \cdot y-1\right)^{2}}{2 \sigma_{A}^{2}}\right) d y\right),
\end{aligned}
$$

and the mapping $\left.F\right|_{\rho}(\cdot)$ represents the input-output relations of the MIMO detector between $I_{\mathrm{E}, \mathrm{DET}}$ and $I_{\mathrm{A}, \mathrm{DET}}$ at $\operatorname{SNR} \rho$, i.e.

$$
I_{\mathrm{E}, \mathrm{DET}}=\left.F\right|_{\rho}\left(I_{\mathrm{A}, \mathrm{DET}}\right) \text {. }
$$

The check nodes of the LDPC code have a transfer characteristic given by the following [12]

$$
\begin{aligned}
I_{\mathrm{E}, \mathrm{CND}} & =f_{c}\left(I_{\mathrm{A}, \mathrm{CND}}\right) \\
& \approx 1-\sum_{i=1}^{D_{c}} \rho_{i} \cdot J\left(\left(d_{c, i}-1\right) \cdot J^{-1}\left(1-I_{\mathrm{A}, \mathrm{CND}}\right)\right),
\end{aligned}
$$

where $\rho_{i}$ is the fraction of the check nodes having edge degree $d_{c, i}$, and $D_{c}$ is the number of different check node degrees.

Following the successful decoding (convergence) criterion provided in [12], the degree profile optimization problem can be reduced to the following maximization problem by taking the LDPC code rate $R$ as the objective

$$
\max _{\left\{\lambda_{i}, \rho_{i}\right\}} R_{\text {outer }}=\max _{\left\{\lambda_{i}, \rho_{i}\right\}}\left(1-\frac{\sum_{i=1}^{D_{c}} \rho_{i} / d_{c, i}}{\sum_{i=1}^{D_{v}} \lambda_{i} / d_{v, i}}\right),
$$

under linear constraints given by

$$
\begin{aligned}
& I_{\mathrm{E}, \mathrm{VND}}\left(I_{\mathrm{A}, \mathrm{VND}}\right) \geq I_{\mathrm{A}, \mathrm{CND}}\left(I_{\mathrm{E}, \mathrm{CND}}\right)=I_{\mathrm{A}, \mathrm{CND}}\left(I_{\mathrm{A}, \mathrm{VND}}\right), \\
& \sum_{i=1}^{D_{v}} \lambda_{i}=1, \quad \sum_{i=1}^{D_{c}} \rho_{i}=1, \quad 0 \leq \lambda_{i}, \rho_{i} \leq 1
\end{aligned}
$$

Utilizing the closed form EXIT functions of the component soft decoders given by (23) and (26), we propose an efficient
LDPC code degree profile optimization algorithm in the following, which is composed of two simple linear optimization steps.

- Variable node degree profile optimization:

For a fixed check node degree profile $\left\{\rho_{i}^{k}\right\}$ from the $k^{\text {th }}$ iteration, the optimal variable node degree profile $\left\{\lambda_{i}^{k+1}\right\}$ is given by

under the constraints

$$
\left\{\lambda_{i}^{k+1}\right\}=\arg \max _{\left\{\lambda_{i}\right\}} \sum_{i=1}^{D_{v}} \lambda_{i} / d_{v, i},
$$

$$
\begin{aligned}
& f_{s}\left(f_{c}\left(a_{n}\right)\right) \geq a_{n}, \\
& \sum_{i=1}^{D_{v}} \lambda_{i}=1, \quad 0 \leq \lambda_{i} \leq 1, \quad 1 \leq n \leq N,
\end{aligned}
$$

where $\left\{a_{n} \mid a_{n} \in[0,1]\right\}$ is a set of specified constraint points, and $N$ is the total number of constraints on the curve.

\section{- Check node degree profile optimization:}

For a fixed variable node degree profile $\left\{\lambda_{i}^{k+1}\right\}$ from the $(k+$ $1)^{t h}$ iteration, the optimal check node degree profile $\left\{\rho_{i}^{k+1}\right\}$ is given by

under the constraints

$$
\left\{\rho_{i}^{k+1}\right\}=\arg \min _{\left\{\rho_{i}\right\}} \sum_{i=1}^{D_{c}} \rho_{i} / d_{c, i},
$$

$$
\begin{aligned}
& f_{c}\left(f_{s}\left(a_{n}\right)\right) \geq a_{n}, \\
& \sum_{i=1}^{D_{v}} \rho_{i}=1, \quad 0 \leq \rho_{i} \leq 1, \quad 1 \leq n \leq N,
\end{aligned}
$$

where $a_{n}$ and $N$ are defined as before.

\section{- Initializations:}

In general, we can start with any feasible degree profiles. Based on our experience from numerical simulations, we find that it is always a good choice to start with a regular check node degree $d_{c}$.

If we stack the LDPC code degree profile $\left\{\lambda_{i}, \rho_{i}\right\}$ into a super vector $\eta=\left[\lambda_{1}, \cdots, \lambda_{D_{v}}, \rho_{1}, \cdots, \rho_{D_{c}}\right]^{T}$. We can see that the objective $R_{\text {outer }}$ given in equation (27) is a concave function with respect to $\eta$ and that all the constraints given in (28) are linear. Hence, the above degree optimization problem has only one unique optimal solution. Due to the non-decreasing property of the proposed iterative maximization algorithm, it is guaranteed to converge to the global maximum solution $\eta^{\star}$ from any initialization point. Therefore, in contrast to the suboptimal manual curving fitting technique proposed in [10], the above iterative LDPC optimization algorithm provides much better performance and can serve as an efficient tool for coded MIMO system design.

\section{Numerical and Simulation Results}

The probability of bit error of a $2 \times 2$ MIMO system over unknown fading channel with coherence time $T=6$, training number $T_{\tau}=2$, and BPSK modulation is demonstrated in Fig. 3. The outer LDPC code is a regular $(3,6)$ code with code rate $R=1 / 2$, and codeword length $8 \times 10^{4}$. As can be 


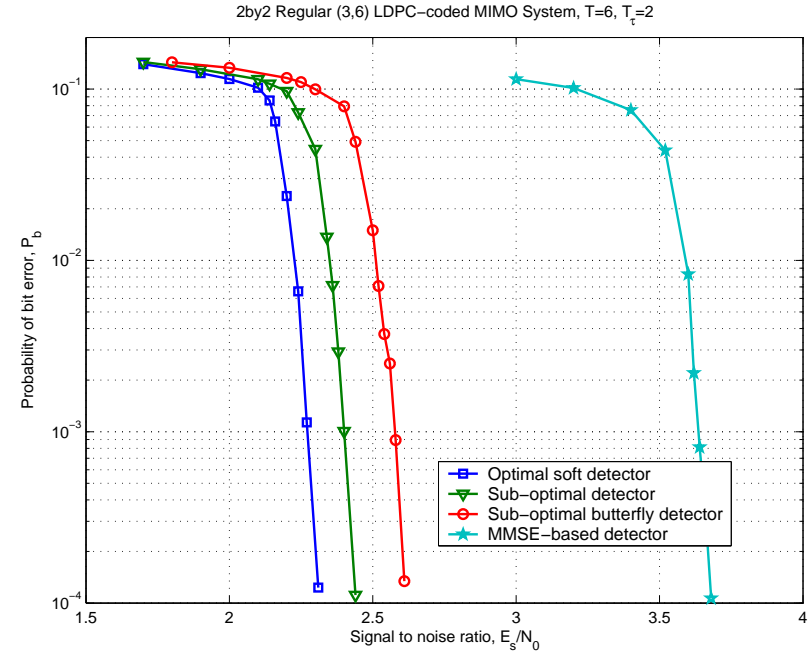

Fig. 3. Probability of bit error of a $2 \times 2$ regular $(3,6)$ LDPC-coded MIMO system over a unknown fading channel with coherence time $T=6$ and training number $T_{\tau}=2$ using several different soft MIMO detectors.

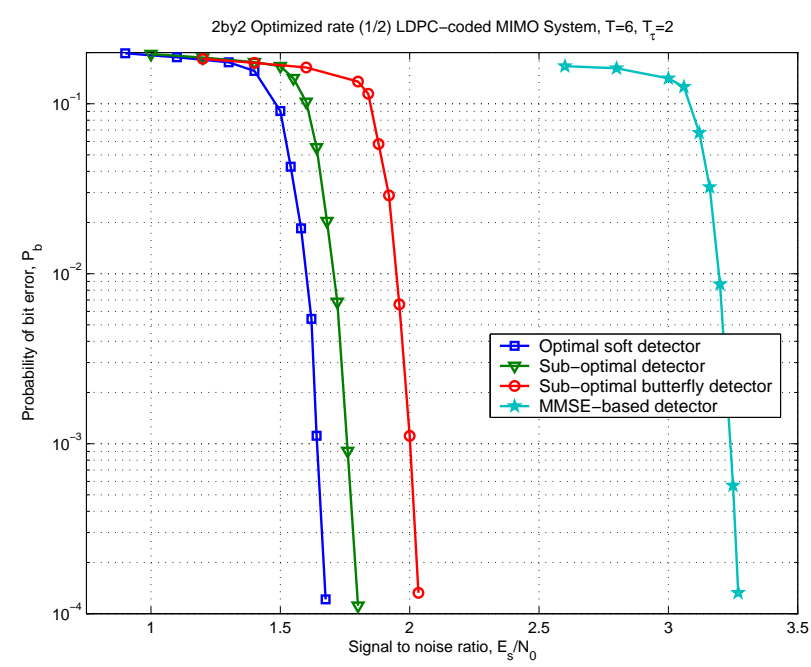

Fig. 4. Probability of bit error of a $2 \times 2$ optimized LDPC-coded MIMO system over a unknown fading channel with coherence time $T=6$ and training number $T_{\tau}=2$ using several different soft MIMO detectors.

observed from the above plot, over $1.5 \mathrm{~dB}$ performance gain can be achieved by using optimal soft MIMO detectors rather than the simple MMSE-based detector. The two sub-optimal MIMO detectors provide significant performance gain, and at the same time maintain affordable decoding complexity.

Using the optimization algorithm provided in Section IV-B, the optimal LDPC code degree profiles (with outer code rate $R_{\text {outer }}=1 / 2$ ) for the coded MIMO system using the different soft MIMO detection algorithms are obtained and used in the overall performance simulation. We consider the same $2 \times 2$ coded MIMO system used in Fig. 3 that transmits over the same unknown fading channel with coherence time $T=6$ and pilot number $T_{\tau}=2$ for simulations. The probability of bit error of the LDPC-coded MIMO system with optimized
LDPC code degree profile is shown in Fig. 4. Compared with Fig. 3, we can achieve about $0.6 \mathrm{~dB}$ performance gain by using the optimized LDPC degree profile as opposed to the simple regular $(3,6)$ LDPC code.

\section{CONCLUSION}

In this paper, we developed a practical LDPC-coded MIMO system over a flat fading wireless environment with no channel state information neither at the transmitter nor at the receiver. We first proposed several soft MIMO detectors, including one optimal soft MIMO detectors and two simplified suboptimal detectors, that offer an effective tradeoff between complexity and performance. A coded MIMO receiver is constructed in an unconventional manner, where the soft MIMO detector and LDPC variable node decoder form one super soft-decoding unit, and the LDPC check node decoder forms the other component of the iterative decoding scheme. By exploiting the proposed receiver structure, tractable extrinsic information transfer functions of the component soft decoders are obtained. Based on the closed form EXIT functions, a simple and efficient LDPC code degree profile optimization algorithm is proposed. The proposed optimization algorithm is shown to have global optimality and guaranteed convergence from any initialization, which is superior to the sub-optimal manual curve fitting technique in previous work. Numerical and simulation results of the unknown LDPC-coded MIMO system using the optimized degree profile further confirm the advantage of using the proposed design approach for the coded MIMO system.

\section{REFERENCES}

[1] E. Telatar, "Capacity of multi-antenna Gaussian channels," European Trans. Telecomm. (ETT), vol. 10, no. 6, pp. 585-595, Nov 1999.

[2] J. H. Kotecha and A. M. Sayeed, "Transmit signal design for optimal estimation of correlated MIMO channels," IEEE Trans. on Signal Processing, vol. 52, pp. 546-557, Feb 2004.

[3] D. Samardzija and N. Mandayam, "Pilot-assisted estimation of MIMO fading channel response and achievable data rates," IEEE Trans. on Signal Processing, vol. 51, pp. 2882-2890, Nov 2003.

[4] H. Sahlin and H. Broman, "MIMO signal separation for FIR channels: A criterion and performance analysis," IEEE Trans. on Signal Processing, vol. 48, pp. 642-649, Mar 2000.

[5] S. Amari and J. F. Cardoso, "Blind source separation-semiparametric statistical approach," IEEE Trans. on Signal Processing, vol. 45, pp. 2692-2700, Nov 1997.

[6] B. L. Hughes, "Differential space-time modulation," IEEE Trans. on Information Theory, vol. 46, pp. 2567-2578, Nov 2000.

[7] B. M. Hochwald and W. Sweldens, "Differential unitary space-time modulation," IEEE Trans. on Communications, vol. 48, pp. 2041-2052, Dec 2000

[8] B. Hochwald and T. Marzetta, "Unitary space-time modulation for multiple-antenna communications in Rayleigh flat fading," IEEE Trans. on Information Theory, vol. 46, pp. 543-564, Mar 2000.

[9] B. M. Hochwald, T. L. Marzetta, T. J. Richardson, W. Sweldens, and R. Urbanke, "Systematic design of unitary space-time constellations," IEEE Trans. on Information Theory, vol. 46, pp. 1962-1973, Sept 2000

[10] S. ten Brink, G. Kramer, and A. Ashikhmin, "Design of low-density parity-check codes for modulation and detection," IEEE Trans. on Communications, vol. 52, pp. 670-678, Apr 2004.

[11] T. L. Marzetta and B. M. Hochwald, "Capacity of a mobile multipleantenna communication link in Rayleigh flat fading," IEEE Trans. on Information Theory, vol. 45, pp. 139-157, Jan 1999.

[12] S. ten Brink, "Convergence behavior of iteratively decoded parallel concatenated codes," IEEE Trans. on Communications, vol. 49, pp. 1727-1737, Oct 2001. 